Gut, 1964, 5, 363

\title{
Superior mesenteric embolectomy
}

\author{
G. L. BOHN AND K. J. CARROLL \\ From the Royal Berkshire Hospital, Reading
}

EDITORIAL SYNOPSIS This paper reports a successful superior mesenteric embolectomy. The possibility of successful surgical treatment makes its recognition particularly important. In any patient with a known history of cardiovascular disease who presents a sudden severe abdominal pain of obscure origin this diagnosis must be entertained. The pain at first is colicky because of the severe peristalsis which is induced but when ischaemia is marked it then becomes constant. With the crises of pain in the early stages the blood pressure falls and then recovers with the cessation of pain and this intermittent shock was a feature in the patient reported. A plain radiograph of the abdomen in the early stages is characterized by the absence or minimal presence of air in both the small and large bowel giving the film a very clear appearance. Prompt exploration is needed.

The first case of a superior mesenteric artery embolectomy with survival of the patient was reported by Shaw and Rutledge in 1957. Since then successes have been reported by Miller and DiMare (1958), Stewart, Sweetman, Westphal, and Wise (1960) and Saris and Uricchio (1960), and recently by Hardy (1963).

Because of the rarity of successful surgical treatment, it has been decided to report another successful case.

\section{CASE REPORT}

The patient, a man of 64 , was admitted as an emergency at 7.30 p.m. on 27 November 1962, with abdominal pain of three and a half hours' duration. The pain was sudden in onset, central abdominal in position, and was of a continuous nature, although he noticed periodic exacerbations. He also said that he was relatively well between the severe episodes of pain and that before an exacerbation he became cold and clammy and started to sweat. His general practitioner also noted in his letter that during the episodes of pain the blood pressure dropped but recovered after the violent pains ceased. The pain went through to his back but did not radiate to shoulders or legs. There was anorexia, nausea, and on two occasions he had vomited black material. His bowels had opened twice, but the motions were of normal colour and consistency. Micturition was normal, and there had been no chest pain or shortness of breath. He smoked 20 cigarettes a day, and had always had a cough.

Electrocardiograms had proved myocardial infarction in 1959 and 1961. There was a three-year history of intermittent claudication, for which he had a bilateral lumbar sympathectomy in 1960, and an achilles tenotomy

${ }^{1}$ Present address: St. Bartholomew's Hospital, London, E.C.1. in 1961. In November 1961 a systolic bruit had been heard over the terminal aorta, iliac and femoral vessels.

On examination he was in pain and sweating. Blood pressure was $200 / 100 \mathrm{~mm}$. $\mathrm{Hg}$; pulse 64 regular; the apex beat was not felt; heart sounds were normal. The abdomen was scaphoid and tender all over, but there was no muscle spasm. Bowel sounds were present and a bruit could be heard on auscultation over the abdominal aorta. The right femoral artery pulse was present but no other pulses could be felt in either limb. Rectal examination revealed no melaena or fresh blood. During the following half hour the patient was re-examined and it was noted that intermittent shock was a feature of his condition. and a provisional diagnosis of superior mesenteric artery thrombosis was made, and the patient prepared for immediate operation.

Laparotomy (G.L.B.) was performed 45 minutes later. A localized block, about $\frac{1}{2}$ in. long, was found in the superior mesenteric artery, just below the upper jejunal branches. The artery above the block was vigorously pulsating. The segment of bowel supplied by the vessel was pale and ischaemic, with no pulsation in the mesenteric vessels and no peristalsis in the bowel itself. There were large calcified mesenteric glands. The aorta was very heavily calcified and there were no aneurysmal dilatations. The colon was normal. The artery was controlled above and below the block, and arteriotomy performed over the block itself. As soon as this was done a piece of clot shot out into the air followed by brisk arterial haemorrhage from the incision. This was closed by a $5 / 0$ silk suture and immediately the distal artery began to pulsate vigorously, as did the smaller vessels in the mesentery near the bowel previously ischaemic.

Post-operatively the patient was given antibiotics but no anticoagulants. He ran a low-grade fever for three days and antibiotics were stopped on the fifth post-operative day. The pulse rate rose to 90 on the third post-operative day and then settled. His bowels opened normally on the 
fourth post-operative day and the stools were normal in colour and consistency. He was discharged on the fourteenth post-operative day and since then has been seen in the follow-up clinic and remains well.

\section{DISCUSSION}

Sudden occlusion of the superior mesenteric artery may be due either to thrombosis or to embolism. Thrombosis develops in relation to atheroma, whilst embolism arises from the fibrillating left auricle, a myocardial infarction, or an atheromatous plaque. It presents as an acute abdominal emergency, and because of the paucity of physical signs in the early stages the patient is often kept under observation and operation delayed.

In any patient with a known history of cardiovascular disease who presents with sudden severe abdominal pain of obscure origin, this diagnosis must be entertained. The pain at first is colicky because of the severe peristalsis which is induced, but when ischaemia is marked it becomes constant. Blood may be vomited or passed in the motion. If there is much blood loss into the bowel wall and lumen shock may be manifest.

The abdominal findings in the early stages may simply amount to some generalized tenderness as in this case, with no distension or muscle spasm.
Sometimes even tenderness is absent. One could therefore say that, like angina pectoris, the diagnosis may rest on the history alone. As ischaemic necrosis progresses, the physical signs become more marked, and the white cell count also rises as a result of necrosis of tissues.

A plain film of the abdomen in the early stages is characterized by an absence or minimal amount of air in both the small and large bowel. The film has thus a very clear appearance. It would be regarded as an ideal preparation for intravenous pyelography. At a later stage, when ileus occurs, distended gas-filled loops will be seen.

We wish to thank the patient's doctor, Dr. N. S. Gent, for prompt referral of the patient to the hospital, and Mr. G. Weston for permission to publish hospital records.

\section{REFERENCES}

Hardy, E. G. (1963). Superior mesenteric embolectomy. Brit. med. J., $1,1658$.

Miller, H. I., and DiMare, S. A. (1958). Mesenteric infarction. New Engl. J. Med., 259, 512-515.

Saris, D. S., and Uricchio, J. F. (1960). Superior mesenteric arteria embolectomy. Arch. Surg., 81, 90-93.

Shaw, R. S., and Rutledge, R. H. (1957). Superior-mesenteric-artery embolectomy in the treatment of massive mesenteric infarction. New Engl. J. Med., 257, 595-598.

Stewart, G. D., Sweetman, W. R., Westphal, K., and Wise, R. A. (1960). Superior mesenteric artery embolectomy. Ann. Surg., 151, 274-278. 